BMC

Genomics

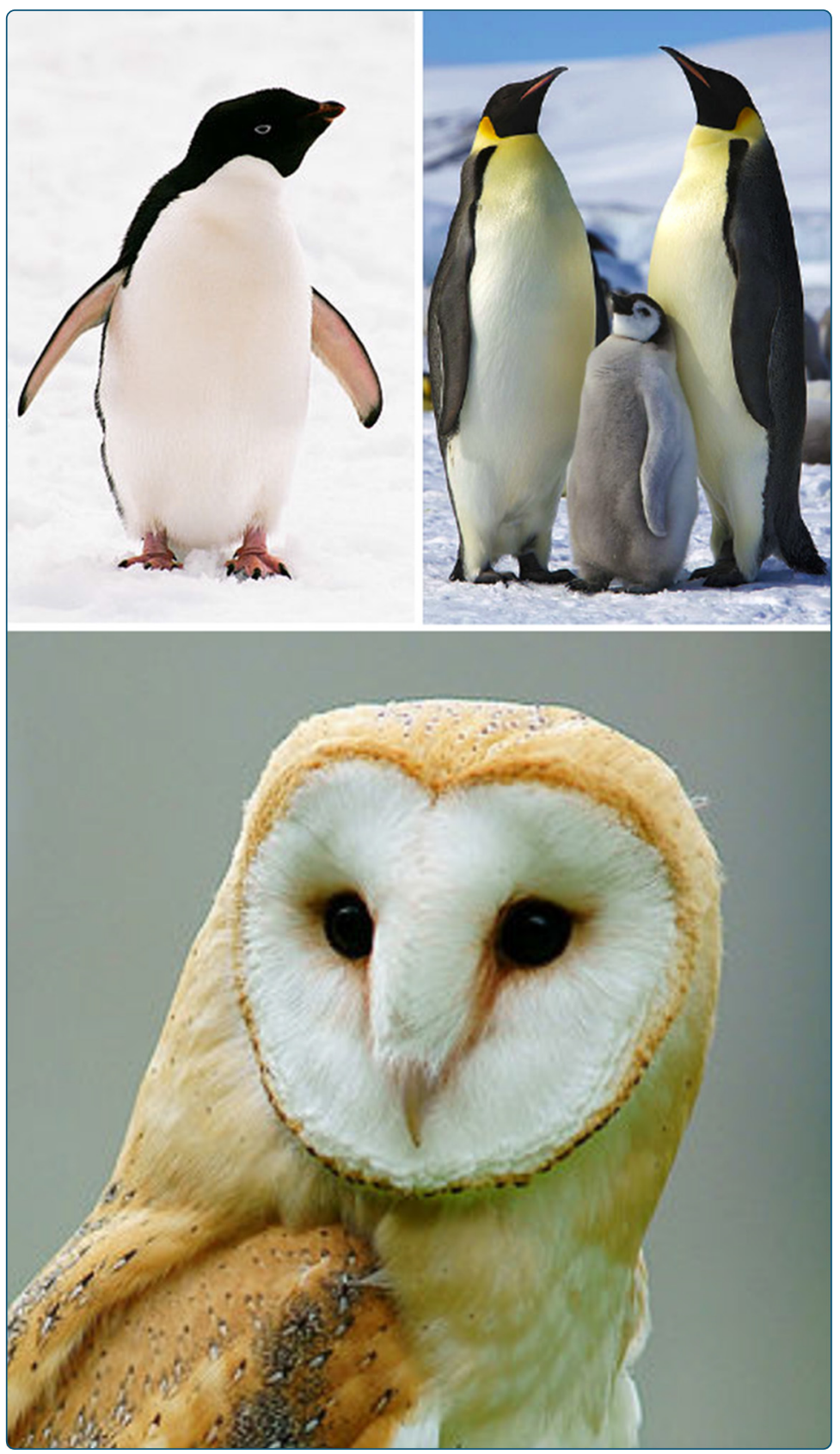

Gene loss, adaptive evolution and the co-evolution of plumage coloration genes with opsins in birds

Borges et al. 


\title{
Gene loss, adaptive evolution and the co-evolution of plumage coloration genes with opsins in birds
}

\author{
Rui Borges ${ }^{1,2}$, Imran Khan ${ }^{1,2}$, Warren E. Johnson ${ }^{3}$, M. Thomas P. Gilbert ${ }^{4}$, Guojie Zhang ${ }^{5,6}$, Erich D. Jarvis ${ }^{7,8}$, \\ Stephen J. O'Brien ${ }^{9,10}$ and Agostinho Antunes ${ }^{1,2^{*}}$
}

\begin{abstract}
Background: The wide range of complex photic systems observed in birds exemplifies one of their key evolutionary adaptions, a well-developed visual system. However, genomic approaches have yet to be used to disentangle the evolutionary mechanisms that govern evolution of avian visual systems.

Results: We performed comparative genomic analyses across 48 avian genomes that span extant bird phylogenetic diversity to assess evolutionary changes in the 17 representatives of the opsin gene family and five plumage coloration genes. Our analyses suggest modern birds have maintained a repertoire of up to 15 opsins. Synteny analyses indicate that PARA and PARIE pineal opsins were lost, probably in conjunction with the degeneration of the parietal organ. Eleven of the 15 avian opsins evolved in a non-neutral pattern, confirming the adaptive importance of vision in birds. Visual conopsins sw1, sw2 and Iw evolved under negative selection, while the dim-light RH1 photopigment diversified. The evolutionary patterns of sw1 and of violet/ultraviolet sensitivity in birds suggest that avian ancestors had violet-sensitive vision. Additionally, we demonstrate an adaptive association between the RH2 opsin and the MC1R plumage color gene, suggesting that plumage coloration has been photic mediated. At the intra-avian level we observed some unique adaptive patterns. For example, barn owl showed early signs of pseudogenization in $\mathrm{RH}$, perhaps in response to nocturnal behavior, and penguins had amino acid deletions in $R H 2$ sites responsible for the red shift and retinal binding. These patterns in the barn owl and penguins were convergent with adaptive strategies in nocturnal and aquatic mammals, respectively.
\end{abstract}

Conclusions: We conclude that birds have evolved diverse opsin adaptations through gene loss, adaptive selection and coevolution with plumage coloration, and that differentiated selective patterns at the species level suggest novel photic pressures to influence evolutionary patterns of more-recent lineages.

Keywords: Opsin, Vision, Birds, Gene loss, Pseudogenization, Co-evolution, Plumage coloration

\section{Background}

Birds are highly visual animals, with a variety of special adaptations to diverse light stimuli [1-3]. Anatomical, physiological, genetic and paleontological evidence suggests that birds rely heavily on visual cues in most aspects of their life history [1]. The bird's eye occupies around $50 \%$ of the cranial volume while retaining the

\footnotetext{
* Correspondence: aantunes@ciimar.up.pt

${ }^{1}$ CIIMAR/CIMAR, Interdisciplinary Centre of Marine and Environmental

Research, University of Porto, Rua dos Bragas, 177, 4050-123 Porto, Portugal

${ }^{2}$ Department of Biology, Faculty of Sciences, University of Porto, Rua do

Campo Alegre, 4169-007 Porto, Portugal

Full list of author information is available at the end of the article
}

same general structure as other vertebrates [1]. The eye has an ellipsoid conformation, a sclerotic ring that integrates a large visual field and a specialized retina that provides high focal acuity [1]. Birds possess cone photoreceptors that are distributed densely over the retina (i.e. multiple foveas) [2] and generally possess a tetrachromatic visual system, with colored oil droplets containing a high concentration of carotenoids that are associated with cone cells $[1,3]$, acting as filters that enhances color discrimination [4]. In contrast, most mammals have two types of cone opsins and are thus generally dichromatic [3]. 
Avian eye complexity has led to a complex array of ecological visual specializations, including use of ultra-violet sensitivity (UVS) [5]. Birds employ UVS variation broadly in recognition of coloration patterns, social signaling, hunting, nectar localization and mate-choice [6-8]. Color cues are also important in avian intra-specific and inter-specific communication, including for evaluating the quality of potential mates (sexual selection), select resources, spot elusive prey and detect predators. Furthermore, birds can use photic stimuli in spatio-temporal orientation. For example, some species use a refined sense of photoperiodicity and day length to assist in magnetic compass orientation [9] and regulate seasonal behaviors including sexual periods and seasonal migratory patterns [10]. Although photoreception is a crucial and ubiquitous trait of birds, our understanding of the co-evolution of behaviors and patterns of genetic variation underlying avian photoreception remains poor.

Birds share with all vertebrates a general mechanism of photoreception that is mediated by opsins, a group of hepta-transmembrane proteins [11] involved in the conversion of a photon of light into an electrochemical signal. Vertebrate opsins have been phylogenetically classified into five subfamilies: (1) the visual opsins, including rhodopsin (RH1) and conopsins (RH2, OPN1sw1, OPN1sw2 and OPN1lw); (2) the melanopsins consisting of two paralogous genes (OPN4m and OPN4x); (3) the pineal subfamily consisting of the parapinopsin (PARA), parietopsin (PARE), pinopsin $(P I N)$ and vertebrate ancient (VA) opsins; (4) the vertebrate non-visual subfamily including encephalopsin (OPN3) and the teleost multiple tissue opsins (TMT and TMT2); and (5) a photoisomerases group including the $R G R, R R H$ and neuropsin (OPN5) genes [11, 12]. These last three groups are referred sensu lato as the nonvisual opsins, as they are involved in non-image forming responses to light [13].

In a preliminary companion study, we found that opsin genes show evidence of having evolved under strong stabilizing selection in birds, with mean $\omega$ values below 0.25 [14]. However, some episodes of positive selection were identified in the $\mathrm{RH} 2$ and OPN1sw1 opsins on the emerging branches of penguins and Passerida [14]. Among the visual opsins, OPN1sw1 plays a role in avian sensitivity to violet (VS) or UVS light, with evidence of positive selection in Passerida probably related with the spectral tuning change from UVS-VS. These shifts can be explained by single nucleotide substitutions at the 86 and 90 spectral tuning sites found in the partial sequence of OPN1sw1 across birds in a past study [15]. Similarly, there is phylogenetic evidence suggesting that avian color vision has shifted between VS and UVS at least 14 times within avian evolution [16].
Despite efforts to better understand the evolution of opsin in birds, most of these studies have focused on visual opsins, and PCR generated fragments of the genes. However, non-visual responses are as important as visual for the overall light adaptive response, and a more complete study is needed to better elucidate the biological details of avian photic adaptation. Here we advanced our findings by performing a comparative analyses across 48 avian species, most recently sequenced [14, 17], to characterize gene gain/loss and the selective forces that have occurred in the 17 vertebrate representatives of the opsin gene family. Our findings provide insight into the history of avian photic adaptation and their coevolving systems.

\section{Results}

\section{Genomic identification of avian opsins}

We retrieved sequences of opsin genes through t-blastn searches in 48 bird genomes [17] using the well annotated chicken (Gallus gallus) and zebra finch (Taeniopygia guttata) opsin gene sequences as queries. We found most opsins assumed to be present in tetrapoda genomes: RH1, RH2, OPN1lw, OPN1sw1, OPN1sw2, OPN4m, OPN4x, OPN3, RGR, RRH, OPN5, PIN and VA (Fig. 1). We also identified for the first time the two different types of teleost multiple tissue opsin genes in birds (designated as TMT and TMT2). We could not find the parietopsin and parapinopsin pineal opsins (PARA and PARIE) in any of the 48 studied genomes (blast searches conducted on the raw read sequences; Fig. 1). Syntenic analyses of other genes around where PARA and PARIE pineal genes were expected suggest that they were lost in birds and mammals (Fig. 2); only non-avian reptiles have the PARIE and PARA genes. These results suggest that birds have a repertoire of 15 opsin representatives, and that PARIE and PARA were independently lost in both birds and mammals.

The presence/absence patterns of opsins on the avian phylogenetic (evolutionary) tree [17] suggests no clear patterns of gene loss (or gain) among avian orders (Fig. 1). This is evidence that no major early events of gene loss (and gain) have occurred during the radiation of the avian lineages and evidence that the avian ancestor had 15 opsin representatives. This also supports the supposition that the tetrachromatic condition (lineages in which we were able to identify the RH2, sw1, sw2 and $l w$ visual conopsins) was the ancestral condition of modern birds.

RH1 and RH2 were present in all the bird genomes, while the $s w 1, s w 2$ and $l w$ conopsins were identified in 41, 31 and 26 of the 48 genomes, respectively (Fig. 1). Even in the high-coverage sequenced genomes $(\geq 80 \mathrm{X})$ we were unable to identify $s w 1$ in Anna's hummingbird 


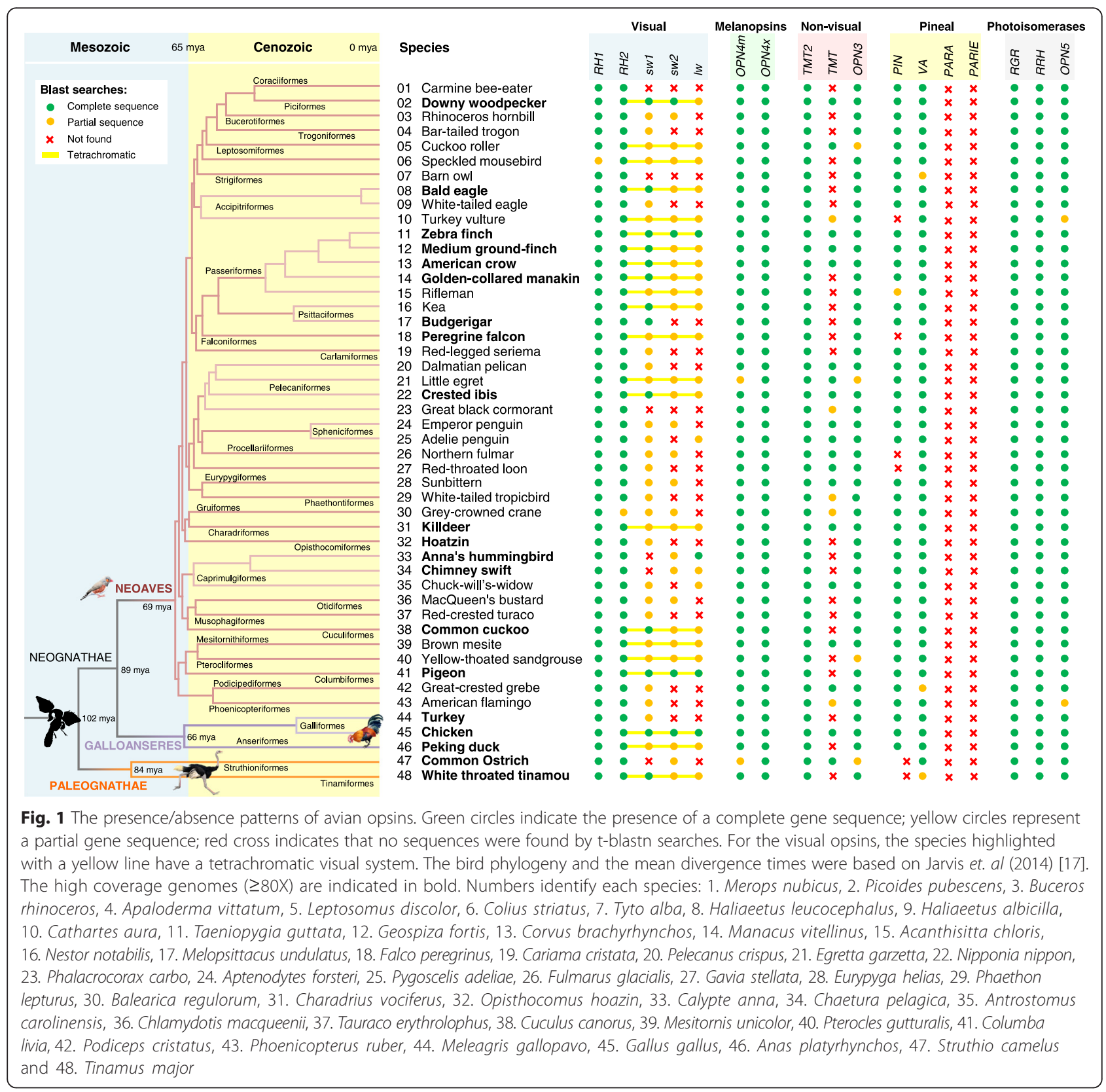

(Calypte anna) and the closely related chimney swift (Chaetura pelagica), $l w$ and $s w 2$ in hoatzin (Opisthocomus hoazin) and $l w$ in the ostrich (Strutio camelus). However, experimental studies have shown that the ostrich possesses four types of cones [18] and $s w 1$ has been previously described in other Trochilidae (humingbirds) [19]. This suggests that some of these absences could be due to the inability to sequence these genes.

To assess if $s w 1, s w 2$ and $l w$ conopsins could be difficult to sequence genes, we assessed the GC (guaninecytosine dinucleotide) content in all opsin genes using the GC ratio method (Additional file 1), which quantifies the relative abundance of the $\mathrm{GC}$ dinucleotide considering the abundance of each of the $\mathrm{C}$ and $\mathrm{G}$ nucleotides in the nucleotide sequence [20]. GC rich regions are known to be more difficult to sequence [21]. We found significant differences in the $\log \mathrm{GC}$ ratio between opsin genes (ANOVA $\mathrm{F}=77.67$ on 14 and 559 degrees of freedom, $p$-value $<0.001)$, with $s w 1, s w 2$ and $l w$ conopsins having significantly higher GC ratios compared with all the other opsins (post-hoc pairwise comparisons, Additional file 2). This finding suggests that $s w 1, s w 2$ and $l w$ conopsins are located in GC-rich regions (such as the microchromosomes), which are known to be more difficult to sequence. 


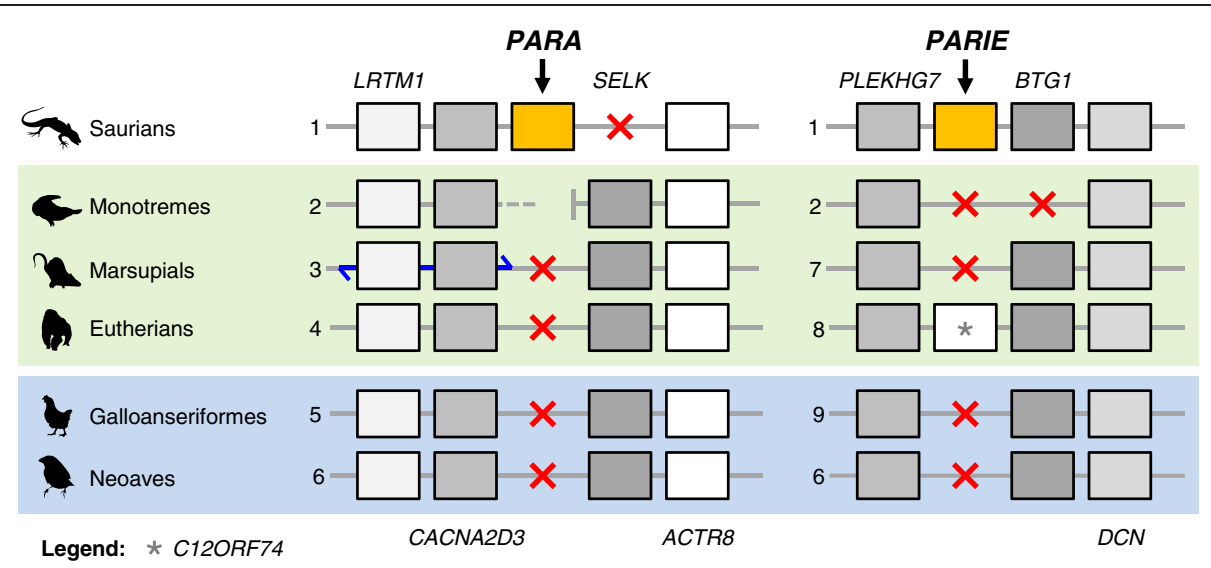

Fig. 2 Syntenic patterns of the PARA and PARIE pineal opsins in mammals and birds. For each species we list the genes adjacent to PARA and PARIE and indicate when they are absent in genome sequences with a red cross. Blue arrow indicates that the region experienced an inversion. The numbers identify each species: 1. Carolina anole (Anolis carolinensis), 2. Platypus (Ornithorhynchus anatinus), 3. Tasmanian devil (Sarcophilus harrisii), 4. Human (Homo sapiens), 5. Chicken (Gallus gallus), 6. Zebra finch (Taeniopygia guttata), 7. Opossum (Monodelphis domestica), 8. African elephant (Loxodonta africana) and 9. Turkey (Meleagris gallopavo)

\section{Site-selection in avian and mammalian opsins}

Using sequences retrieved from the 48 avian genomes we conducted phylogenetic and selection analyses. From the codon-based alignments and using the species phylogenetic tree (Fig. 1; [17]), we estimated the $\omega$-ratio (the ratio between the non-synonymous by synonymous rate of substitutions) as an indicator of selective pressures acting on protein-coding genes [22]. Six of the 15 avian opsins, RH1, PIN, VA, RGR, RRH and OPN4x, showed evidence of positive selection (Table 1 and Additional file 3) while five, OPN1sw1, OPN1sw2, $O P N 1 l w, T M T$ and OPN3, showed evidence of negative selection. RH2, TMT2, OPN5 and OPN4m evolved neutrally. We found statistical evidence $(>0.95$ posterior probability) that $217 \mathrm{~A}$ in $R H 1$ was positively selected (G. gallus amino acid number based on bovine rhodopsin amino acid numeration). We performed phylogenetic reconstruction of the 217 site using the well resolved avian species tree [17]. We found that $217 \mathrm{~T} / \mathrm{M} / \mathrm{A}$ residues are associated with the evolution of neoaves in water and land environments (Additional file 4). The land bird clades recurrently evolved the $M$ residue, while the water bird clades evolved the T/A residues.

To test whether these changes are specific to birds, or present more broadly, we performed site-selection tests models on mammalian opsins. We found mammals possess 4 opsins evolving under positive selection ( $s w 1$, $O P N 3, R R H$ and OPN4m) and only one evolving through negative selection (RH1) (Table 1 and Additional file 3 ). We compared the $\omega$-ratios in mammals and birds using the estimated $\omega$-site categories ( $\omega \mathrm{B}$ and $\omega \mathrm{M})$ and their proportions under the statistically significant site-selection model. We used the bootstrap technique (10,000 replicates) to estimate the expected value of the probability of a sampled $\omega \mathrm{B}$ category being higher than a sampled $\omega \mathrm{M}$ category $(p)$. The expected values of $p$ and the respective odd scores $(>2.5)$ indicate that mammals evolved with higher adaptive rates at the

Table 1 Site-selection tests for the avian and mammalian opsins

\begin{tabular}{lllll}
\hline Gene & $\omega \mathrm{B}$ & $\omega \mathrm{M}$ & $\mathrm{E}[p]$ & Odd score \\
\hline RH1 & $0.061^{* *}$ & $0.064^{*}$ & 0.411 & 1.429 \\
RH2 & 0.080 & & & \\
OPN1sw1 & $0.041^{*}$ & $0.178^{* *}$ & 0.261 & 2.822 \\
OPN1sw2 & $0.050^{*}$ & & & \\
OPN1/W & $0.024^{*}$ & 0.135 & 0.215 & 3.654 \\
TMT & $0.220^{*}$ & & & \\
TMT2 & 0.118 & & & \\
OPN3 & $0.101^{*}$ & $0.310^{* *}$ & 0.210 & 3.761 \\
PIN & $0.230^{* *}$ & & & \\
VA & $0.265^{* *}$ & & & \\
RGR & $0.148^{* *}$ & 0.193 & 0.316 & 2.166 \\
RRH & $0.155^{* *}$ & $0.273^{* *}$ & 0.333 & 2.000 \\
OPN5 & 0.112 & 0.089 & 0.497 & 1.013 \\
OPN4x & $0.171^{* *}$ & & & \\
OPN4m & 0.203 & $0.239^{* *}$ & 0.470 & 1.128 \\
\hline W valesw & & & \\
\hline
\end{tabular}

$\omega$ values were calculated using the $\omega$ categories and the respective proportions under the statistcally significant site-selection model: $\left({ }^{* *}\right)$ positive selection, $\left({ }^{*}\right)$ negative selection or neutral evolution (unmarked). $p$ is the probability of a sampled $\omega B$ category being higher than a sampled $\omega M$ category. $E[p]$ is the expected value of $p$ using the bootstrap technique for 100000 bootstraps. The odd score is the $(1-p) / p$ ratio and indicates the likelihood $\omega M$ categories are higher than the $\omega \mathrm{B}$ categories 
sites in $s w 1, l w$ and $O P N 3(2.82,3.65,3.77$ odd score, respectively; Table 1).

\section{Species-specific branch selection}

Branch-specific selection models implemented in PAML [22] were used to estimate the foreground evolutionary rate of each opsin gene in each of the studied species $(\omega$-lineage) (Additional file 5). We implemented a phylogenetic logistic regression between the VS and UVS condition in each avian species and the respective $\omega$-lineage value. Ödeen et al. (2009) have validated the use of genomic DNA to predict the VS and UVS subtypes in $s w 1$ opsins [23]. All the amino acid patterns we found in the 84-94 region of our sequences were already described in the literature $[15,16,24]$, thus providing confirmation of VS/UVS inferences (Additional material 6). The boxplots (Fig. 3a) depict that $s w 1 \omega$-lineage values are strongly correlated with the VS/UVS condition in birds, with the accelerated lineages being the moststrongly linked with UVS sensitivity: VS log-odd score $=4.44-79.91 \omega(p$-value $=0.046 ;$ Wald z-statistics, Additional file 6). The VS log-odd score corresponds to the logarithm of the ratio between the probability of a certain lineage to be VS and its contrary (to be UVS) given the $\omega$-lineage. The inferred ancestral condition for the most recent common ancestor of birds $(\omega$-lineage $=0$ ) was one of increased VS sensitivity (as opposed to UVS), with a 4.44 log-odd score (i.e. with $98.8 \%$ of probability).
In addition, the golden-collared manakin (Manacus vitellinus) and the American crow (Corvus brachyrhynchos) Passeriformes and the tinamou (Tinamus major) showed a relative higher $\omega$-lineage for the VS-type (Fig. 3a).

We used principal component analysis with an outlier map to assess which lineages had unique patterns in opsins based on their $\omega$-tendencies. The most informative principal components revealed prominent clusters consistent with the inferences gained from the phylogenetic hierarchy (i.e. closely related species were plotted nearby - results not shown). However, three species were clear outliers: the Adélie and emperor penguins (Pygoscelis adeliae and Aptenodytes forsteri) and the barn owl (Tyto alba) (Fig. 3b).

Sequence analysis of the barn owl RH2 opsin, had several non-synonymous mutations in very conserved regions of the gene and indels and a stop codon at position 168 where the $\mathrm{W}$ residue is present in other all birds (Additional file 7). In penguins, the $\mathrm{RH} 2$ sequence has a segmental deletion in the S295-S298 region. In addition, the PIN sequence in Adélie penguin had evidence of pseudogenization, with a frame-shift alteration and a stop codon. Li et al. (2014) suggested that there was a pseudogenization event for PIN in both penguins and reported several stop codons and frame shift alterations [25]. Although these features were found in the Adélie penguin, they were not observed in the emperor penguin PIN sequence (Afo_R013563). Further
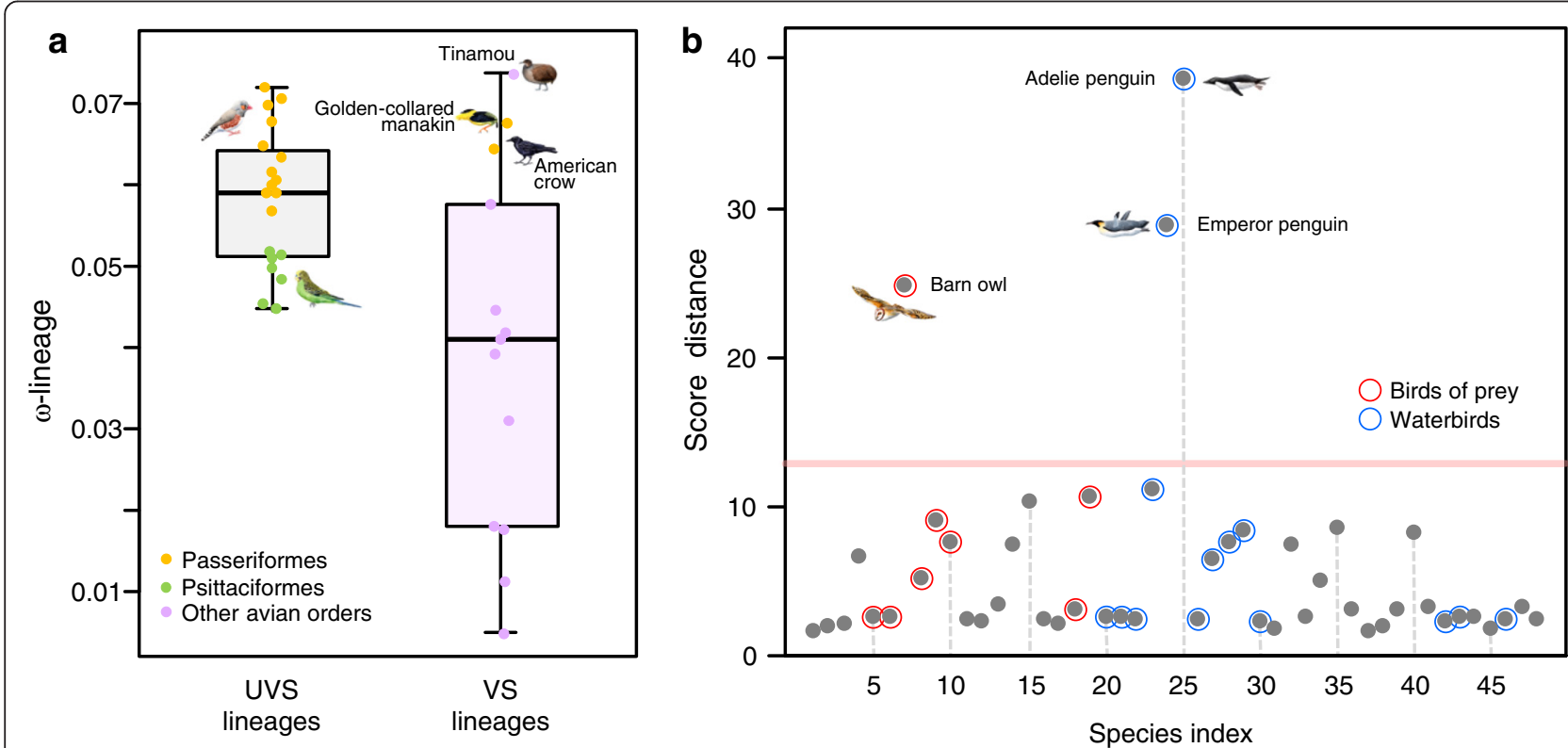

Fig. 3 Lineage-specific visual adaptations in birds. a Box plots summarizing the lineage-specific evolutionary rates in bird species that possessed a violet (VS) or ultra-violet (UVS) sws1 opsin. Colored circles identify the observed $\omega$-lineage for Passeriformes (yellow) and Psittaciformes (green). b Distance plot indicating the lineages with an outlier evolutionary behavior in the opsin gene family. Species highlighted with red circumferences are birds of prey and those in blue are water birds. The species numbering system is the same as in Fig. 1 
inspection of the penguins $R H 1$ amino acid sequences revealed a site-specific variation in the 194 tuning site [26]: $\mathrm{L} \rightarrow \mathrm{P}$.

We additionally implemented branch-specific tests on the terminal lineage of the barn owl and the stem lineage of penguins to test whether the $\omega$-ratio on these branches $(\omega \mathrm{f})$ have differentiated adaptive behavior (Additional file 8): in the barn owl lineage we found a significant accelerated $\omega$-branch for PIN $(\omega \mathrm{f} / \omega \mathrm{b}=0.548 / 0.146)$; in penguins, RH1 (0.119/0.043), OPN4x (0.546/0.155), PIN $(0.527 / 0.146)$ and OPN5 $(0.408 / 0.107)$ also had a significantly accelerated $\omega f$. Due to the early signs of pseudogenization in the barn owl $R H 2$ and the emperor penguin PIN opsins, we did not implement branch-specific tests for those lineages. We have also excluded the TMT and VA opsins from branch-tests of the in the barn owl lineages because only partial sequences were available (Fig. 1).

\section{Opsin vs. melanin-based coloration genes co-evolution analyses}

In order to assess if the evolution of photoreception in birds is related with the evolution of plumage coloration, we also analyzed the melanin-based plumage coloration genes: ASIP, OCA2, TYR, TYRP1 and MC1R [27]. These genes are part of the biochemical cascade leading to melanin production, which is, along with the carotenoids, responsible for pigmentation in birds [28, 29]. Site-selection analyses have shown that $M C 1 R$ evolved under negative selection, while evidence of positive selection was found in the OCA2, TYR and ASIP (Additional material 3).

To test for gene-evolution associations, we calculated the species-specific $\omega$-lineage for the melanin-based plumage coloration genes, as we did for the avian opsin genes.
We classified the $\omega$-lineages into three evolutionary categories: accelerated (more than 0.75 quartile), conserved (less than the 0.25 quartile) and neutral, and implemented association tests for each of the visualcoloration gene pairs. The expected proportion of lineages with the same evolutionary category under independence was $6 / 16$ (0.375), which was tested against the alternative hypothesis that $p>6 / 16$. We found a significant association ( $p$-value $<8.4 \mathrm{e}-4$, Bonferroni corrected for multiple tests) between $M C 1 R$ and $R H 2$ (Fig. 4; we excluded the OPN1sws 2 and OPN1lw opsins due to insufficient sampling size for implementing the association tests $(\leq 15))$. The statistical parameters of the co-evolution analysis are in Additional file 9. These associations are not related by physical location in the genome, as relative syntenic location analyses show that the associations are physically independent of genome location (Additional file 10).

\section{Discussion}

We believe that our study is among the most comprehensive genomic analyses of opsins in vertebrates, and particularly in birds. We have been able to characterize losses, gains, and selective evolution that are correlated with lineage-specific traits. Below we discuss the implications of the key highlights for each family of opsins.

\section{The loss of PARA and PARIE in mammals and birds}

The apparent independent loss of PARA and PARIE in mammals and birds is intriguing. In non-avian and nonmammalian vertebrates PARA and PARIE pineal opsins genes are expressed in the parietal organ, which is a part of the epithalamus [30]. The parietal organ, along with the pineal organ, forms the parietal eye (or third eye),

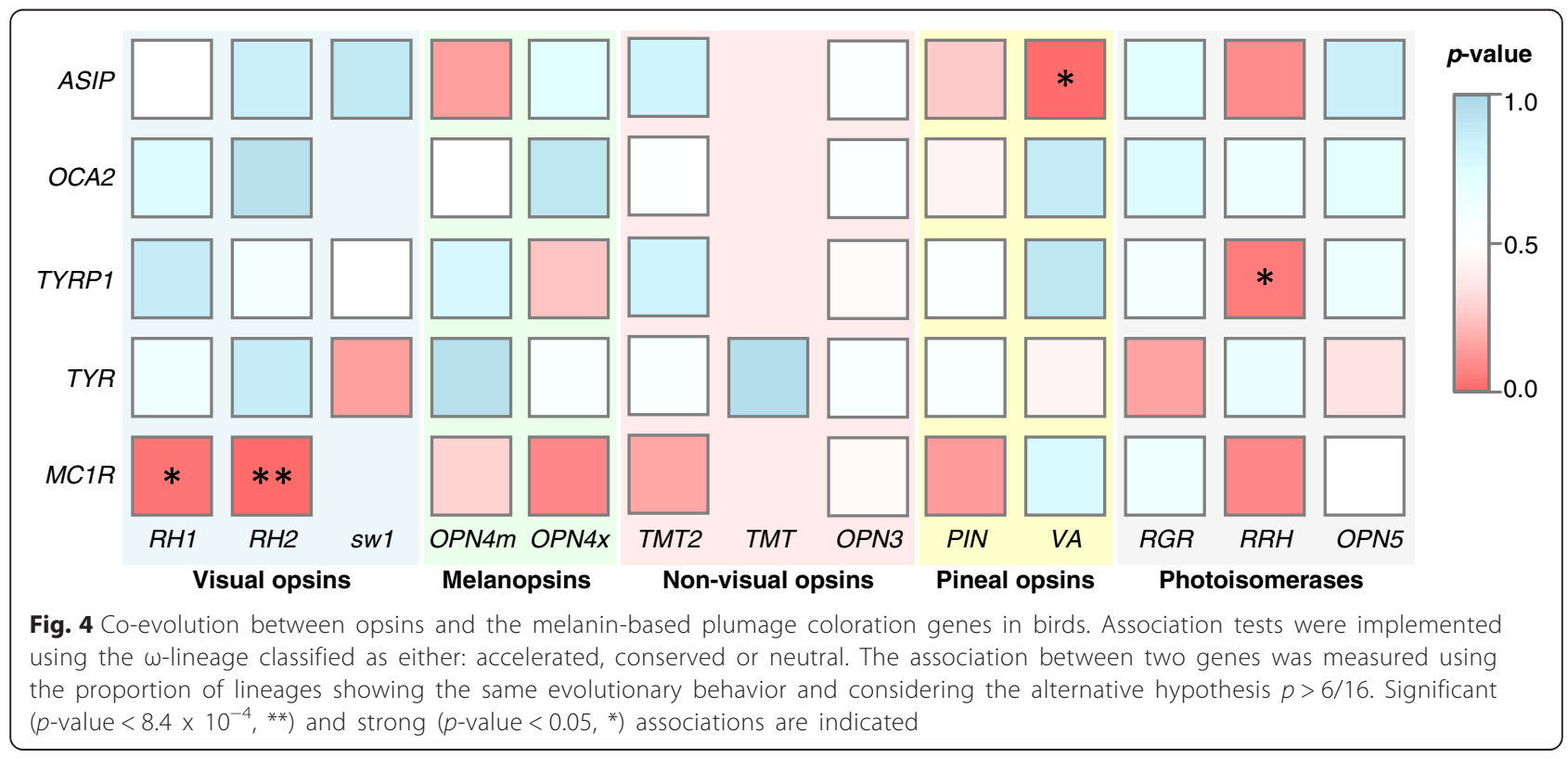


which functions as a proper photoreception organ, regulating circadian rhythmicity and hormone production for thermoregulation [30]. In birds and mammals the parietal organ degenerates completely $[12,31,32]$, which is very likely associated with the loss of PARA and PARIE. Taking into consideration the thermoregulatory function of the parietal organ in vertebrates [30] and that mammals and birds are endothermic, we consider that the independent loss of these genes in these lineages could be related with a change in the mechanisms regulating body temperature. As mammals and birds became less reliant on external sources of energy to maintain body temperature (evolution of endothermy [33]), the parietal organ would have degenerated (accompanied by the related signaling pathways).

\section{Adaptive evolution of avian opsins}

Apart from the PARA and PARIE opsins, most modern birds maintained the vertebrate repertoire of opsins, suggesting that birds never became specifically adapted to limited photic conditions that might have led to the extensive pseudogenization of opsins. Additionally, since we find no global events of opsin loss during the early modern avian species radiation, birds appeared to have possessed tetrachromatic vision (RH2, OPN1sw1, OPN1sw2 and OPN1lw) for most of their evolutionary history. This suggests that birds relied on a visual system specialized for discriminating different light qualities, particularly useful in complex photopic environments, where birds likely diversified.

In birds, 11 of the 15 avian opsins evolved in a nonneutral manner: RH1, OPN1sw1, OPN1sw2, OPN1lw, TMT, OPN3, PIN, VA, RGR, RRH and OPN4x. This suggests that visual and non-visual adaptive strategies have been imperative during avian evolution, validating the importance of the visual sense in birds. Among the visual opsins, $s w 1, s w 2$ and $l w$ have been more conserved in birds, while for $R H 1$ positive selection was found in birds and negative selection in mammals. Visual conopsins perform image-forming functions [34], and are particularly important for photopic animals, like birds. The patterns of purifying evolution in the $s w 1, s w 2$ and $l w$ would have permitted fine adjustment of the spectral sensitivities of these opsins, ensuring elevated photic acuity throughout avian evolution. In addition, the higher site-specific adaptive rates of $s w 1$ and $l w$ opsins in mammals relative to birds is consistent with our preliminary analyses in Zhang et al. (2014) [14], where we have found that the $s w 1 \omega$-ratio is lower in birds than in mammals $(0.16 / 0.21$ respectively). This suggests that the mechanism to maintain optimal color discrimination is more stringent in birds than in mammals.

RH1 has the key function of conferring monochromatic vision in low light environments [35], and thus it is not surprising that it diversifies in birds that were mostly photopic-adapted. In contrast, in mammals the negative selection is consistent with their nocturnal habits and the anatomical features of the mammalian eye that are congruent with nocturnal ancestry [36]. More specifically, our findings that site 217 of $R H 1$ evolved under positive selection in birds is consistent with findings of positive-selection on this site in other vertebrates [26, 37]; however, it has been reported that different amino acids at these sites do not seem to cause spectral shifts in $R H 1$ [26]. Instead, we found an association between the $\mathrm{T}$ and M/A amino acid residues and the evolution of land and water neoaves clades - similar ecological condition also influenced the evolution of the olfactory receptor subgenomes [38]. Possible contributions of these specific amino acid substitutions to water and land adaptations can be tested through in-vitro experiments to verify the role of this site in $R H 1$ spectral tuning. Overall, the contrasting evolutionary signatures in the visual opsins between mammals and birds are consistent with their contrasting photic needs in that scotopic-adapted animals need to maximize the amount of light collected, while photopic-adapted animals require enhanced visual acuity.

\section{OPN1sw1 evolution and VS/UVS vision}

Our analysis suggesting that the ancestral bird possessed a VS OPN1sw1 opsin is consistent with other analyses of amino acid variation of $s w 1$ spectral tuning sites that concluded that VS was the probable ancestral condition of birds [16, 39]. The ecological role of the OPN1sw1 opsin in birds is not well understood, but is likely to be broad, as it has been associated with coloration pattern recognition [19], social signaling, hunting, nectar localization and mate-choice [6-8]. Others also suggest a role in non-visual processes such as circadian rhythm regulation $[40,41]$. Species in six avian orders, Pteroclidiformes, Charadriiformes, Coraciiformes, Trogoniformes, Psittaciformes and Passeriformes, have been shown to possess UVS sensitivity [16], of which the later four belong to core landbirds. However, there are no clear patterns of coloration, breeding behaviors, activity patterns and feeding habits among the species in these groups that would explain the acquisition of UVS (or the retention of VS).

The use of UVS is most clear among Passeriforms, which is consistent with evidence of strong positive selection found in the branch leading to the passerine group for the sw1 gene [14]. However, some Passeriformes species are also VS, which is reflected in a relative higher $\omega$-lineage in the golden-collared manakin and American crow. These two species have been reported as cases of recent adaptation to the VS vision [42]. The contrasting root-to-tip $\omega$ lineages appears to be an efficient methodology to study 
ecological adaptation scenarios in phylogenetic contexts, as it is sensitive enough to detect episodes of reversal evolution.

Another species associated with a high $\omega$-lineage for the VS class is the paleognath tinamou. The tinamou sw1 amino acid sequences had F, C and $\mathrm{M}$ residues at sites 86, 90 and 93, which corresponds with the amino acid conformation found by Ödeen et al. (2013) in the closely-related ostrich (Struthio camelus) [16]. The sw1 sensitivity in paleognaths has been somewhat controversial, because while microspectrophotometry analysis of the $s w 1$ in ostrich suggests VS [18], the $86 \mathrm{~F}$ and $90 \mathrm{C}$ residues support UVS [41]. Our results, linking the tinamou with a high $\omega$-lineage in the VS class suggests that the UVS $\rightarrow$ VS shift was relatively recent. This is congruent with the amino acid sequence similarity with UVS sw1 and also with the microspectrophotometry analysis. Therefore, we presume that there are other amino acids in addition to 86 and 90 that likely changed the spectral sensitivity of the $s w 1$ photopigment, such as $93 \mathrm{M}$ as suggested by Ödeen et al. (2013) [16].

\section{MC1R/RH2 co-evolution}

The adaptive association between the $M C 1 R$ gene and the $R H 2$ visual opsin in avian species suggest that plumage colorations have been photic mediated. The MC1R receptor is involved in melanin-based coloration in vertebrates and is found primarily in melanocyte cells where it controls the deposition of melanin in tissues [43]. Activation of $M C 1 R$ leads to increased synthesis of black/brown eumelanin, whereas low MC1R activity leads to increased synthesis of red/yellow phaeomelanin [44]. First cloned from chickens [45], several studies have found that $M C 1 R$ is closely associated with plumage coloration [46-49]. MC1R adaptive evolution has also been correlated with the degree of sexual dichromatism in galliform birds, suggesting that MC1R may be a key link in the interaction of sexual selection and plumage colour [50]. Sexual selection would also be a reasonable explanation for the $M C 1 R / R H 2$ co-evolution. Indeed, one would expect that plumage coloration patterns would only be important for birds if they were associated with a visual system capable of "read" plumage coloration cues. Indeed, it has been shown that tetrachromatic vision, a process which requires the $\mathrm{RH} 2$ photopigment, enhances plumage discriminability in birds [51]. In addition, Bloch et al. (2015) have suggested that rapid evolution of $\mathrm{RH} 2$ in Setophaga birds (a genus of Passeriformes) is linked to sexual selection, given their exceptional plumage color diversification [52]. In situations in which sexual selection evolves in association with plumage coloration patterns and color discriminability, then there should be strong associations among $M C 1 R$ and visual opsins. Further tests on the $s w 1, s w 2$ and $l w$ opsins would be welcome.

\section{More-recent photic adaptations in birds: the barn owl}

The barn owl has very distinctive photoreceptive features relative to other birds [53, 54]. Due to a recent nocturnal adaptation, barn owls have frontally placed eyes and anatomical adaptations that improve perception of photic stimuli in low light environments; this includes an elongated eye and a high ratio between the eye and corporal sizes $[53,54]$. The pseudogenization of $\mathrm{RH} 2$ and the lineage-specific acceleration of $P I N$ are consistent with these adaptive changes.

$\mathrm{RH} 2$ is sensitive to the green photo spectrum from about 480-535 $\mathrm{nm}$ [2] and has undergone rapid gene loss and gain in other vertebrate lineages (reviewed in [55]). In addition, $R H 2$ was lost in placental mammals during the nocturnal bottleneck $[3,36]$. There is evidence that some owls have a photoreceptor that is sensitive to the $503 \mathrm{~nm}$ spectrum, which would be consistent with a RH2-type photopigment [56]. If confirmed that this photopigment is from $R H 2$, the pseudogenization event reported here for the barn owl would likely be lineage-specific.

$P I N$ is a blue-sensitive pigment $(\sim 470 \mathrm{~nm})$ expressed in the pineal gland (determined in chicken) that has a role controlling the circadian pacemaker and the rhythmic production of melatonin $[57,58]$. The accelerated evolutionary rate observed in the barn owl PIN is appreciable $(0.548 / 0.146$, i.e. 3.7 times faster than the avian trend) and includes several non-synonymous mutations. Although it is not known if the PIN opsin is fully functional in the barn owl pineal gland, owls possess a rudimentary pineal with the pinealocytes having rudimentary photoreceptive features [59].The PIN protein may continue to have a role in circadian tasks associated with a nocturnal lifestyle or the degeneration of the pineal gland may have permitted the unconstrained molecular evolution of PIN.

Other genes that are likely to be involved in the adaptation of birds to a nocturnal life style include $s w 1, s w 2$ and $l w$ visual conopsins, which we were not found in the barn owl genome. Zhao et al. (2009a) performed phylogenetic analysis in the $l w$ and $s w 1$ photopigments in nocturnal bats and affirmed the importance of the $s w 1$ in the species' sensory ecology [60]. In particular, it would be important to determine if the $s w 1$ photopigment in birds is UV/UVS sensitive, as UVS vision has been associated with nocturnal habits in mammals [60, 61].

\section{More-recent photic adaptations in birds: the penguins} Penguins possess specialized and unique optic adaptations, including an approximately-spherical lens and a flat cornea that augment their vision when underwater 
[62]. At the molecular level, evidence of gene pseudogenization and positive selection in phototransduction genes have been associated with the aquatic lifestyle of the Adélie and emperor Antarctic penguins [25]. Penguin specializations include cone visual pigments tuned towards the blue-green range of the visual spectrum, presumably related with the spectral composition of their aquatic environment [63]. The retuning of the $\mathrm{RH} 2$ in penguins could be linked with non-synonymous mutations in the D83, Q122, A164, A207 and S222 amino acids of $R H 2$ (site identification based on bovine rhodopsin homolog sites) [55, 64]. Although we did not find any variation in the two penguins for these sites, we found site deletions at S295 and K296. S295 is responsible for the red shift in RH2 [65] while $296 \mathrm{~K}$ is an important retinal binding site [14]. It is not known if this causes $R H 2$ to be non-functional in penguins, which would require functional experimental tests. However, these indels were shared by the two penguin species, which shared a common ancestor 23.0 mya [17], meaning that if they were deleterious (or significantly compromise the $R H 2$ function) we would expect the $R H 2$ to pseudogenize over that time period, which was not observed in any of the penguin lineages. Most likely, these indels are evidence that penguins have adapted a unique mechanism to perform the molecular interactions that mediate the photon absorption in $\mathrm{RH} 2$ that is better suited for underwater environments.

Additionally, we have found evidence of accelerated evolution of RH1, OPN4X and OPN5 in the penguin lineage. $O P N 4 x$ is only present in non-mammalian vertebrates and is associated with non-image-forming light responses, including circadian entrainment [66-68]. The accelerated evolution of OPN4x suggests that penguins may have evolved new circadian responses to cope with the seasonal particularities of Antarctica, including the dramatic daily light changes and hourly differences. OPN5 is UV-sensitive, is expressed in the chicken retina and pineal gland, and plays a role in the assistance of an 11-cis-retinal-supplying system [69]. The role of the OPN5 in penguin vision is less obvious.

RH1 has been associated with nocturnal/diurnal terrestrial lifestyles, but some studies have shown that $R H 1$ underwent shifts in spectral tuning in marine mammals [70]. Evidence from aquatic mammals are in congruence with the accelerated evolution of RH1 in the ancestral lineage leading to penguins. Zhao et al. (2009b) [71] reported evidence of positive selection in the cetacean and pinniped aquatic clades, suggesting that RH1 evolution were related with the turbid condition of aquatic environments. In addition, the occurrence of $\mathrm{P}$ in the 194 spectral tunning site was also verified in cetaceans, particularly in the sowerby's beaked whale (Mesoplodon bidens) [71]. Changes in RH1 molecular features are compelling evidence that it contributed to penguin's unique adaptive strategies to aquatic environments.

Similarly as for the barn owl, the emperor penguin also showed lineage-specific changes in PIN. However, we were unable to determine if is this is a case of adaptive or unconstrained evolution. Nevertheless, evidence suggests that the pineal organ of the Antarctic penguin (Pygoscelis papua) lacks typical photoreceptor elements [72], which as observed by Li et al. (2014), is the likely cause of the accelerated evolution/pseudogenization of the PIN opsin in penguins [25].

\section{Conclusion}

The analyses of visual and non-visual opsins from 48 genomes spanning the extant avian phylogeny provide new insights on the evolutionary history of avian visual systems by revealing the molecular signatures that characterize their evolution. Our results suggest that avian adaptive strategies were driven mainly by gene loss, adaptive (negative and positive) selection and coevolution with melanin-based plumage coloration genes. More-recent evolutionary events in the owl and penguins lineages suggest the emergence of new adaptive strategies among birds, probably promoted by the evolution of the nocturnal and aquatic lifestyles, respectively. We conclude that birds, while being remarkably dependent on the visual sense, have changed their visual and nonvisual molecular systems in response to the photic environment they occupy and to the strong pressures of sexual selection.

\section{Methods}

\section{Phylogenetic analysis}

Psi-blast and t-blastn searches were performed employing annotated protein sequences of the zebrafinch and chicken opsin gene in the NCBI, Ensembl data bases $[73,74]$ and in 45 avian genomes from the Avian Phylogenomics Project $[17,75]$. Additionally, plumage coloration gene sequences from the ASIP, MC1R, TYR, $T Y R P 1$ and OCA 2 genes were also obtained from these databases. Accession numbers for all the sequences used in this study are compiled in Additional files 6, 11, 12 and 13.

A protein-based coding sequence alignment was performed by aligning the translated sequences using the Muscle 3.3 algorithm [76] and subsequently improved manually -gap rich and ambiguous regions were removed. Partial sequences were excluded from phylogenetic analysis. The presence of saturation in base substitution for each of the alignments was tested using DAMBE5 [20]. None of the alignments showed evidence of saturation bias (Additional file 14) and thus, all were acceptable for phylogenetic analysis. jModelTest (version 0.1.1) with Akaike Information criterion (AIC) was used to estimate 
the most appropriate nucleotide substitution model [77]. For all genes the GTR $+\mathrm{I}+\Gamma$ model was selected as the most-appropriate model with $95 \%$ confidence.

\section{Site- and branch-specific selection analyses}

Opsin gene alignments and the species tree were used in the codeml package in PAML 4.4 software [78] to assess the site- and branch-specific codon substitution models of maximum likelihood. The genome-scale avian species tree [17] was used to perform the site and branchspecific selection analysis because we aim to trace gene evolution within a framework of species evolution and to eliminate possible confounding effects of gene tree phylogeny variation or error. The site specific models were tested comparatively: M7 (beta) vs M8 (beta $+\omega$ ) and M8a (beta $+\omega=1$ ) vs M8 [22]. Subsequently, likelihood rate comparisons (LRT) were performed to test which models best fit the data. M7 and M8 assume a $\beta$ distribution for the $\omega$ value between 0 and 1 but M8 additionally allows the occurrence of positively-selected sites $(\omega>1)$. M8a tests for neutral evolution by fixing the parameter $\omega=1$. Whenever the likelihood ratio test was significant under the M8 model, the Bayes Empirical Bayes (BEB) method was used to calculate posterior probabilities of the sites that are subject to positive selection (accepted at $>0.95$ ) [79]. Site-selection analysis were implemented in PAML4 package [78].

The branch selection models were implemented comparing the estimated $\omega$-ratio for all lineages in the tree (one-ratio model) and the two-ratios model, which assigns two $\omega$ ratios for the foreground and background branches [80]. The foreground $\omega$-lineage maximum likelihood estimation was calculated for each species of each gene labeling the tip, the root and all intermediate branches. For the species-specific branch analysis on the barn owl terminal lineage and the emerging lineage of penguins, we test the significance of the $\omega$ variations (one-ratio $v s$. two-ratio model with 1 degree of freedom [80]) using the LRT to perform the hypothesis testing.

\section{Statistical analysis}

We implemented a phylogenetic logistic regression that explains the VS or UVS $s w 1$ sensitivity in each species using the $\omega$-lineage using phylolm package in $\mathrm{R}$ software [81]. Additionally, a robust principal component analysis of $\omega$-lineage values was conducted following the Hubert (2005) approach [82], in order to assess the avian opsin outlier map, which summarizes those lineages that show unexpected $\omega$-tendencies in the evolutionary dimensional space of the studied opsins.

To seek evidence for opsin and plumage coloration gene co-evolution we used the $\omega$-lineage foreground rate of evolution, classified in three evolutionary categories: accelerated (A, more than 0.75 quartile), conserved (C, less than the 0.25 quartile) or neutral ( $\mathrm{N}$, otherwise). Association tests for each visual-coloration gene pairs were implemented considering those lineages that showed the same evolutionary behavior: AA, CC or NN. Since we were interested in these particular 3 coevolving behaviors (amongst nine possibilities), we implemented a proportion test, assuming that the null hypothesis $p=6 / 16$. Under independence, $6 / 16$ is the expected proportion of lineages showing the same evolutionary behavior. Associations with less than 15 comparative pairs were excluded to avoid false positives and the Bonferroni correction was applied for multiple association tests (59 multiple tests, $p$-value $\left.=8.4 \times 10^{-4}\right)$. All statistical analyses were performed using the R software [81].

\section{Availability of supporting data}

The data sets supporting the results of this article are included within the article and its additional files.

\section{Additional files}

\begin{abstract}
Additional file 1: GC ratio comparisons for avian opsins. The $\mathrm{GC}$ ratio corresponds to the $\% \mathrm{GC} /[\% \mathrm{G} * \% \mathrm{C}]$ ratio. GC ratio equal to 1 corresponds to the absence of GC bias, while value lees than 0.76 or higher than 1.25 (horizontal red lines in the plot) indicate deviances in GC use. (PDF $12 \mathrm{~kb}$ )
\end{abstract}

Additional file 2: Log GC ratio pairwise comparisons between the avian opsins. The presented $p$-values are corrected using the Bonferroni adjustment. $p$-values colored in red represent statistically non-significant differences between the average log GC ratios of the compared genes. Pairwise comparisons for the sW1, sw2 and IW opsins are highlighted in grey. (PDF 27 kb)

Additional file 3: Site-selection tests for the avian and mammalian opsins and the melanin-based coloration genes. The logarithm of the model likelihood is represented by InL, the number of model parameters are represented by $n p$ and the LRT is the likelihood ratio test. Accepted site-selection model are indicated with an asterisk $\left(^{*}\right)$ when the M7 model of negative selection is statistically significant, or a double asterisk $\left.{ }^{(* *}\right)$ if the M8 model of positive selection is statically significant. All the LRT comparisons were performed assuming a significance level of 0.05 . (PDF $103 \mathrm{~kb}$ )

Additional file 4: Phylogenetic reconstruction of the RH1 217 residue in birds. The species tree was used as described by Jarvis et al. (2014) [17]. Red and blue species represent land and water birds respectively. Colored ancestral lineages are suggestive of the M (Met; red), $\mathrm{L}$ (Leu; blue) and A (Ala; violet) residues in the 217 site of the $\mathrm{RH} 1$ opsin. (PDF $19 \mathrm{~kb}$ )

Additional file 5: Species-specific $\boldsymbol{\omega}$-lineage estimates. The $\omega$-lineage estimates under the assumption of the branch-specific two-ratios model. Species-specific $\omega$-lineage was calculated considering the species tree and the root-to-tip labeling. (PDF $67 \mathrm{~kb}$ )

Additional file 6: UVS/VS condition for avian sw1 sequences. Avian sW1 sequences used in this study and the respective accession numbers. UVS/UV sw1 type was inferred using the 86, 90 and 93 spectral tuning sites (highlighted in red). Amino acid combinations were compared with previously published sequences in Ödeen et al. (2013) [16]. (PDF 174 kb)

Additional file 7: Amino acid sequence of the barn owl (Tyto alba) RH2 opsin. RH2 conopsin multiple sequence alignment of the barn owl and the zebra finch (Neoaves representative), chicken (Galloanseres representative) and ostrich (Paleognathae representative). Regions in red 
indicate special features of the barn owl RH2 sequence: non-synonymous mutations, indels and stop-codons. (PDF $130 \mathrm{~kb}$ )

Additional file 8: Species-specific branch selection tests. The oneratio model $(\mathrm{HO})$ was tested against the two-ratios model considering the alternative hypotheses of verifying differentiated $\omega$-ratios in the terminal lineage of the barn owl $(\mathrm{H} 1.1)$ or the emerging lineage of penguins $(H 1.2)$. Significant alternative hypothesis are marked with a red $p$-value. InL is the logarithm of the model likelihood and the LRT is the likelihood ratio test. All the LRT comparisons were performed with 1 degree of freedom and assuming a significance level of 0.05. (PDF $147 \mathrm{~kb}$ )

\section{Additional file 9: Co-evolution between opsins and the} melanin-based plumage coloration genes in birds. Association tests were implemented using the $\omega$-lineage classified in three categories: accelerated, conserved and neutral. The association between two genes was measured using the proportion of lineages showing the same evolutionary behavior and considering the alternative hypothesis $p>6 / 16$ Values in the table correspond to the Pearson's $x^{2}$ test statistic and the sample proportions (in brackets). Significant ( $p$-value $<8.4 \times 10^{-4}$ ) and strong $(p$-value $<0.05)$ associations are indicated in red-bold and bold, respectively. (PDF $92 \mathrm{~kb}$ )

Additional file 10: Genomic localization of the genes used for the co-evolution analysis. Genomic localization of the visual genes (in orange) and melanin-based plumage coloration genes (in red) in the chicken and zebra finch karyotypes. (PDF $15 \mathrm{~kb}$ )

Additional file 11: Avian opsin and melanin-based plumage coloration sequences. Accession numbers are indicated for the complete sequences, while for partial sequences the genomic location is given. Asterisk $\left(^{*}\right)$ indicate that we were not able to identify the gene in the respective genome. Gene sequences are available in the Avian Phylogenomics Project database (http://avian.genomics.cn/en/, [75]). (PDF 205 kb)

Additional file 12: Avian sw2 and Iw sequences. Accession number of the sw2 and IW sequences used for site selection analyses. (PDF $12 \mathrm{~kb}$ )

Additional file 13: Mammalian opsin sequences. Accession number of the mammalian opsins sequences used for site selection analyses. (PDF $88 \mathrm{~kb}$ )

Additional file 14: Saturation analysis. The presence of saturation in base substitution for each of the gene alignment was tested by comparing half of the theoretical saturation index expected when assuming full saturation (Iss.c, critical value) with the observed saturation index (Iss). Absence of substitution saturation is verified when Iss is lower than Iss.c for a significant $p$-value. (PDF $24 \mathrm{~kb}$ )

\section{Abbreviations}

AIC: Akaike Information criterion; GC: Guanine-cytosine dinucleotide; LRT: Likelihood ratio test; UVS: Ultra-violet sensitivity; VS: Violet sensitivity.

\section{Competing interests}

The authors declare that they have no competing interests.

\section{Authors' contributions}

RB performed the phylogenetic, evolutionary and structure-function analyses and drafted the manuscript. IK performed the initial gene mining analysis and participated in the drafting of the study. WEJ, MTPG, GZ, EDJ and SJO participated in the drafting and coordination of the study. AA participated in the design, genetic analyses, drafting and coordination of the study. All authors read and approved the final manuscript.

\section{Authors' information}

Not applicable.

\section{Availability of data and materials}

Not applicable.

\section{Acknowledgements}

The authors thank João Paulo Machado and Cidália Gomes for useful discussions during this work. We would also like to thank the anonymous reviewers for constructive comments on an earlier version of this manuscript.

\section{Funding}

RB and IK were funded with a PhD grant from Fundação para a Ciência e a Tecnologia (FCT) (RB:SFRH/BD/79850/2011 and IK: SFRH/BD/48518/2008). EDJ was supported by the Howard Hughes Medicallnstitute. SJO was supported in part by Russian Ministry of Science Mega-grant no.11.G34.31.0068. AA was partially supported by the Strategic Funding UID/Multi/04423/2013 through national funds provided by FCT and European Regional Development Fund (ERDF) in the framework of the programme PT2020, and the FCT projects PTDC/AAC-AMB/121301/2010 (FCOMP-01-0124-FEDER-019490) and PTDC/ AAGGLO/6887/2014.

\section{Author details}

${ }^{1}$ CIIMAR/CIMAR, Interdisciplinary Centre of Marine and Environmental Research, University of Porto, Rua dos Bragas, 177, 4050-123 Porto, Portugal. ${ }^{2}$ Department of Biology, Faculty of Sciences, University of Porto, Rua do Campo Alegre, 4169-007 Porto, Portugal. ${ }^{3}$ Smithsonian Conservation Biology Institute, National Zoological Park, 1500 Remount Road, Front Royal, VA 22630, USA. ${ }^{4}$ Centre for GeoGenetics, Natural History Museum of Denmark, University of Copenhagen, Øster Volgade 5-7, 1350 Copenhagen, Denmark. ${ }^{5}$ China National GeneBank, BGI-Shenzhen, Shenzen 518083, China. ${ }^{6}$ Centre for Social Evolution, Department of Biology, Universitetsparken 15, University of Copenhagen, DK-2100 Copenhagen, Denmark. ${ }^{7}$ Department of Neurobiology, Duke University Medical Center Durham, Box 3209, North Carolina 27710, USA. ${ }^{8}$ Howard Hughes Medical Institute, Chevy Chase, Maryland 20815, USA. ${ }^{9}$ Theodosius Dobzhansky Center for Genome Bioinformatics, St. Petersburg State University, St. Petersburg 199004, Russia. ${ }^{10}$ Oceanographic Center, Nova Southeastern University, 8000 N. Ocean Drive, Ft Lauderdale, Florida 33004, USA.

Received: 14 November 2014 Accepted: 11 September 2015 Published online: 06 October 2015

\section{References}

1. Walls G. The Vertebrate Eye and Its Adaptive Radiation. New York: Hafner publishing company; 1942

2. Hart NS. The visual ecology of avian photoreceptors. Prog Retin Eye Res. 2001:20:675-703.

3. Jacobs $\mathrm{GH}$. Evolution of colour vision in mammals. Philos Trans R Soc Lond B Biol Sci. 2009:364:2957-67.

4. Vorobyev M. Coloured oil droplets enhance colour discrimination. Proc Bio Sci. 2003;270:1255-61.

5. Yokoyama S, Radlwimmer FB, Blow NS. Ultraviolet pigments in birds evolved from violet pigments by a single amino acid change. Proc Natl Acad Sci. 2000;97:7366-71.

6. Bennett ATD, Cuthill IC, Partridge JC, Maier EJ. Ultraviolet vision and mate choice in zebra finches. Nature. 1996;380:433-5.

7. Viitala J, Korplmäki E, Palokangas P, Koivula M. Attraction of kestrels to vole scent marks visible in ultraviolet light. Nature. 1995;373:425-7.

8. Burkhardt D. UV vision: a bird's eye view of feathers. J Comp Physiol A. 1989:164:787-96

9. Wiltschko W, Munro U, Ford H, Wiltschko R. Red light disrupts magnetic orientation of migratory birds. Nature. 1993;364:525-7.

10. Dominoni D, Quetting M, Partecke J. Artificial light at night advances avian reproductive physiology. Proc Biol Sci. 2013;280:20123017.

11. Terakita A. The opsins. Genome Biol. 2005;1:1-9.

12. Peirson S, Halford S, Foster R. The evolution of irradiance detection: melanopsin and the non-visual opsins. Philos Trans R Soc Lond B Biol Sci. 2009:364:2849-65.

13. Shichida Y, Matsuyama T. Evolution of opsins and phototransduction. Philos Trans R Soc Lond B Biol Sci. 2009;364:2881-95.

14. Zhang G, Li C, Li Q, Li B, Larkin DM, Lee C, et al. Comparative genomics reveals insights into avian genome evolution and adaptation. Science. 2014;346:1311-20.

15. Ödeen $\mathrm{A}$, Håstad $\mathrm{O}$. Complex distribution of avian color vision systems revealed by sequencing the SWS1 opsin from total DNA. Mol Biol Evol. 2003;20:855-61.

16. Ödeen $\mathrm{A}$, Håstad $\mathrm{O}$. The phylogenetic distribution of ultraviolet sensitivity in birds. BMC Evol Biol. 2013;13:36.

17. Jarvis ED, Mirarab S, Aberer AJ, Li B, Houde P, Li C, et al. Whole-genome analyses resolve early branches in the tree of life of modern birds. Science. 2014;346:1320-31.

18. Wright MW, Bowmaker JK. Retinal photoreceptors of paleognathous birds: the ostrich (Struthio camelus) and rhea (Rhea americana). Vision Res. 2001:41:1-12. 
19. Ödeen A, Håstad O. Pollinating birds differ in spectral sensitivity. J Comp Physiol A Neuroethol Sensory, Neural, Behav Physiol. 2010;196:91-6.

20. Xia X. DAMBE5: A Comprehensive Software Package for Data Analysis in Molecular Biology and Evolution. Mol Biol Evol. 2013;30:1720-8.

21. Chen Y-C, Liu T, Yu C-H, Chiang T-Y, Hwang C-C. Effects of GC Bias in Next-Generation-Sequencing Data on De Novo Genome Assembly. PLoS One. 2013;8:e62856.

22. Anisimova M, Bielawski JP, Yang Z. Accuracy and power of the likelihood ratio test in detecting adaptive molecular evolution. Mol Biol Evol. 2001;18:1585-92

23. Ödeen A, Hart NS, Håstad O. Assessing the use of genomic DNA as a predictor of the maximum absorbance wavelength of avian SWS1 opsin visual pigments. J Comp Physiol A Neuroethol Sensory, Neural, Behav Physiol. 2009;195:167-73

24. Ödeen A, Håstad O. New primers for the avian SWS1 pigment opsin gene reveal new amino acid configurations in spectral sensitivity tuning sites. J Hered. 2009;100:784-9.

25. Li C, Zhang Y, Li J, Kong L, Hu H, Pan H, et al. Two Antarctic penguin genomes reveal insights into their evolutionary history and molecular changes related to the Antarctic environment. Gigascience. 2014;3:27.

26. Yokoyama S, Tada T, Zhang H, Britt L. Elucidation of phenotypic adaptations: Molecular analyses of dim-light vision proteins in vertebrates. Proc Natl Acad Sci. 2008;105:13480-5

27. Sturm RA. Molecular genetics of human pigmentation diversity. Hum Mol Genet. 2009;18:R9-R17.

28. Mundy NI. Coloration and the Genetics of Adaptation. PLoS Biol. 2007:5:e250.

29. Hoekstra HE, Hirschmann RJ, Bundey RA, Insel PA, Crossland JP. A Single Amino Acid Mutation Contributes to Adaptive Beach Mouse Color Pattern. Science. 2006;313:101-4.

30. Dodt $E$, Meiss $H$. The pineal and parietal organs of lower vertebrates. Experientia. 1982;38:996-1000.

31. Natesan A, Geetha L, Zatz M. Rhythm and soul in the avian pineal. Cell Tissue Res. 2002;309:35-45.

32. Arendt J. Melatonin and the pineal gland: influence on mammalian seasonal and circadian physiology. Rev Reprod. 1998;3:13-22.

33. Grigg GC, Beard LA, Augee ML. The evolution of endothermy and its diversity in mammals and birds. Physiol Biochem Zool. 2004;77:982-97.

34. Okano T, Kojima D, Fukada Y, Shichida Y, Yoshizawa T. Primary structures of chicken cone visual pigments: vertebrate rhodopsins have evolved out of cone visual pigments. Proc Natl Acad Sci. 1992;89:5932-6.

35. Liu J, Liu MY, Nguyen JB, Bhagat A, Mooney V, Yan ECY. Thermal properties of rhodopsin: insight into the molecular mechanism of dim-lingth vision. J Biol Chem. 2011;286:27622-9.

36. Gerkema MP, Davies WIL, Foster RG, Menaker M, Hut RA. The nocturnal bottleneck and the evolution of activity patterns in mammals. Proc Biol Sci. 2013;280:20130508.

37. Sivasundar A, Palumbi SR. Parallel amino acid replacements in the rhodopsins of the rockfishes (Sebastes spp.) associated with shifts in habitat depth. J Evol Biol. 2010;23:1159-69.

38. Khan I, Yang Z, Maldonado E, Li C, Zhang G, Gilbert MTP, et al. Olfactory Receptor Subgenomes Linked with Broad Ecological Adaptations in Sauropsida. Mol Biol Evol 2015:msv155. [Epub ahead of print]

39. Yokoyama S, Shi Y. Genetics and evolution of ultraviolet vision in vertebrates. FEBS Lett. 2000;486:167-72.

40. Zawilska JB, Rosiak J, Nowak JZ. Effects of Near-Ultraviolet (UV-A) Light on Melatonin Biosynthesis in Vertebrate Pineal Gland. Neurosignals. 1999;8:64-9.

41. Aidala Z, Huynen L, Brennan PLR, Musser J, Fidler A, Chong N, et al. Ultraviolet visual sensitivity in three avian lineages: paleognaths, parrots, and passerines. J Comp Physiol A Neuroethol Sens Neural Behav Physiol. 2012;198:495-510.

42. Ödeen A, Håstad O, Alström P. Evolution of ultraviolet vision in the largest avian radiation - the passerines. BMC Evol Biol. 2011;11:313.

43. Mundy NI. A window on the genetics of evolution: MC1R and plumage colouration in birds. Proc Biol Sci. 2005;272:1633-40.

44. Robbins L. Pigmentation phenotypes of variant extension locus alleles result from point mutations that alter MSH receptor function. Cell. 1993;72:827-34.

45. Takeuchi S, Suzuki H, Hirose S, Yabuuchi M, Sato C, Yamamoto H, et al. Molecular cloning and sequence analysis of the chick melanocortin 1-receptor gene. Biochim Biophys Acta - Gene Struct Expr. 1996;1306:122-6.

46. Theron E, Hawkins K, Bermingham E, Ricklefs RE, Mundy NI. The molecular basis of an avian plumage polymorphism in the wild: A melanocortin-1- receptor point mutation is perfectly associated with the melanic plumage morph of the bananaquit, Coereba flaveola. Curr Biol. 2001;11:550-7.

47. Uy JAC, Moyle RG, Filardi CE, Cheviron ZA. Difference in plumage color used in species recognition between incipient species is linked to a single amino acid substitution in the melanocortin-1 receptor. Am Nat. 2009;174:244-54.

48. Doucet SM, Shawkey MD, Rathburn MK, Mays HL, Montgomerie R. Concordant evolution of plumage colour, feather microstructure and a melanocortin receptor gene between mainland and island populations of a fairy-wren. Proc Biol Sci. 2004;271:1663-70.

49. Nadeau NJ, Minvielle F, Mundy NI. Association of a Glu92Lys substitution in MC1R with extended brown in Japanese quail (Coturnix japonica). Anim Genet. 2006;37:287-9.

50. Nadeau NJ, Burke T, Mundy NI. Evolution of an avian pigmentation gene correlates with a measure of sexual selection. Proc Biol Sci. 2007:274:1807-13.

51. Vorobyev M, Osorio D, Bennetta TD, Marshall NJ, Cuthill IC. Tetrachromacy, oil droplets and bird plumage colours. J Comp Physiol. 1998;183:621-33.

52. Bloch NI, Price TD, Chang BSW. Evolutionary dynamics of Rh2 opsins in birds demonstrate an episode of accelerated evolution in the New World warblers (Setophaga). Mol Ecol. 2015;24:2449-62.

53. Van der Willigen RF, Frost B, Wagner H. Stereoscopic depth perception in the owl. Neuroreport. 1998;9:1233-7.

54. Fite KV, Rosenfield-Wessels S. A comparative study of deep avian foveas. Brain Behav Evol. 1975;12:97-115.

55. Bowmaker JK. Evolution of vertebrate visual pigments. Vision Res. 2008;48:2022-41.

56. Bowmaker JK, Martin GR. Visual pigments and colour vision in a nocturnal bird, Strix aluco (tawny owl). Vision Res. 1978;18:1125-30.

57. Okano T, Fukada Y. Photoreception and circadian clock system of the chicken pineal gland. Microsc Res Tech. 2001;53:72-80.

58. Okano T, Yoshizawa T, Fukada Y. Pinopsin is a chicken pineal photoreceptive molecule. Nature. 1994:372:94-7.

59. Haldar C, Guchhait P. Pineal gland of a nocturnal bird, Indian spotted owlet, Athene brama: Morphological and endocrine observations. J Exp Zool. 2000;287:145-50.

60. Zhao H, Rossiter SJ, Teeling EC, Li C, Cotton JA, Zhang S. The evolution of color vision in nocturnal mammals. Proc Natl Acad Sci. 2009:106:8980-5.

61. Peichl L. Diversity of mammalian photoreceptor properties: Adaptations to habitat and lifestyle? Anat Rec Part A Discov Mol Cell Evol Biol. 2005;287A:1001-12.

62. Sivak J, Howland HC, McGill-Harelstad P. Vision of the Humboldt Penguin (Spheniscus humboldti) in Air and Water. Proc R Soc B Biol Sci. 1987;229:467-72

63. Bowmaker JK, Martin GR. Visual pigments and oil droplets in the penguin, Spheniscus humboldti. J Comp Physiol A. 1985;156:71-7.

64. Hunt DM, Carvalho LS, Cowing JA, Davies WL. Evolution and spectral tuning of visual pigments in birds and mammals. Philos Trans R Soc Lond B Biol Sci. 2009:364:2941-55.

65. Heath LA, Wilkie SE, Bowmaker JK, Hunt DM. The rod and green cone opsins of two avian species, the budgerigar, Melopsittacus undulatus, and the mallard duck, Anas platyrhynchus. Gene. 1997;204:121-6.

66. Panda S. Melanopsin Is Required for Non-Image-Forming Photic Responses in Blind Mice. Science. 2003;301:525-7.

67. Bellingham J, Chaurasia SS, Melyan Z, Liu C, Cameron MA, Tarttelin EE, et al. Evolution of melanopsin photoreceptors: discovery and characterization of a new melanopsin in nonmammalian vertebrates. PLoS Biol. 2006:4:e254.

68. Borges R, Johnson WE, O'Brien SJ, Vasconcelos V, Antunes A. The role of gene duplication and unconstrained selective pressures in the melanopsin gene family evolution and vertebrate circadian rhythm regulation. PLoS One. 2012;7:e52413.

69. Yamashita T, Ohuchi H, Tomonari S, Ikeda K, Sakai K, Shichida Y. Opn5 is a UV-sensitive bistable pigment that couples with Gi subtype of $\mathrm{G}$ protein Proc Natl Acad Sci. 2010;107:22084-9.

70. Fasick Jl, Robinson PR. Spectral-tuning mechanisms of marine mammal rhodopsins and correlations with foraging depth. Vis Neurosci. 2000;17:S095252380017511X.

71. Zhao H, Ru B, Teeling E, Faulkes C, Zhang S, Rossiter S. Rhodopsin Molecular Evolution in Mammals Inhabiting Low Light Environments. PLoS One. 2009:4:e8326.

72. Piezzi R, Gutiérrez L. Electron microscopic studies on the pineal organ of the antarctic penguin, (Pygoscelis papua). Cell Tissue Res. 1975;164. 
73. Flicek P, Amode MR, Barrell D, Beal K, Billis K, Brent S, et al. Ensembl 2014. Nucleic Acids Res. 2014;42(Database issue):D749-55.

74. McEntyre J, Ostell J, Pruitt K, Brown G: The Reference Sequence (RefSeq) Database. 2nd Ed 2012:http://www.ncbi.nlm.nih.gov/books/NBK143764/.

75. Zhang G, Li B, Li C, Gilbert M, Jarvis ED, Wang J. Comparative genomic data of the Avian Phylogenomics Project. Gigascience. 2014;3:26.

76. Edgar RC. MUSCLE: multiple sequence alignment with high accuracy and high throughput. Matrix. 2004;32:1792-7.

77. Posada D. jModelTest: phylogenetic model averaging. Mol Biol Evol. 2008;25:1253-6.

78. Yang Z. PAML 4: phylogenetic analysis by maximum likelihood. Mol Biol Evol. 2007;24:1586-91.

79. Yang Z, Wong WSW, Nielsen R. Bayes empirical bayes inference of amino acid sites under positive selection. Mol Biol Evol. 2005;22:1107-18.

80. Yang Z, Nielsen R. Codon-substitution models for detecting molecular adaptation at individual sites along specific lineages. Mol Biol Evol. 2002:19:908-17.

81. R Development Core Team R: R: A Language and Environment for Statistical Computing. R Found Stat Comput 2011:409. [R Foundation for Statistical Computing]

82. Hubert M, Rousseeuw PJ, Vanden Branden K. ROBPCA: A New Approach to Robust Principal Component Analysis. Technometrics. 2005;47:64-79.

\section{Submit your next manuscript to BioMed Central and take full advantage of:}

- Convenient online submission

- Thorough peer review

- No space constraints or color figure charges

- Immediate publication on acceptance

- Inclusion in PubMed, CAS, Scopus and Google Scholar

- Research which is freely available for redistribution 